\title{
Superconformal multi-black hole quantum mechanics
}

\section{Citation}

Michelson, Jeremy, and Andrew Strominger. 1999. "Superconformal Multi-Black Hole Quantum Mechanics." Journal of High Energy Physics 1999 (9): 005-005. https:// doi.org/10.1088/1126-6708/1999/09/005.

\section{Permanent link}

http://nrs.harvard.edu/urn-3:HUL.InstRepos:41417424

\section{Terms of Use}

This article was downloaded from Harvard University's DASH repository, and is made available under the terms and conditions applicable to Other Posted Material, as set forth at http:// nrs.harvard.edu/urn-3:HUL.InstRepos:dash.current.terms-of-use\#LAA

\section{Share Your Story}

The Harvard community has made this article openly available.

Please share how this access benefits you. Submit a story.

Accessibility 


\title{
Superconformal Multi-Black Hole Quantum Mechanics
}

\author{
Jeremy Michelson* \\ Department of Physics \\ University of California \\ Santa Barbara, CA 93106 \\ and \\ Jefferson Physical Laboratories \\ Harvard University \\ Cambridge, MA 02138
}

\section{Andrew Strominger \\ Jefferson Physical Laboratories \\ Harvard University \\ Cambridge, MA 02138}

\begin{abstract}
The quantum mechanics of $N$ slowly-moving charged BPS black holes in five-dimensional $\mathcal{N}=1$ supergravity is considered. The moduli space metric of the $N$ black holes is derived and shown to admit 4 supersymmetries. A near-horizon limit is found in which the dynamics of widely separated black holes decouples from that of strongly-interacting, near-coincident black holes. This decoupling suggests that the quantum states supported in the near-horizon moduli space can be interpreted as internal states of a single composite black hole carrying all of the charge. The near-horizon theory is shown to have an enhanced $D(2,1 ; 0)$ superconformal symmetry. Eigenstates of the Hamiltonian $H$ of the near-horizon theory are ill-defined due to noncompact regions of the moduli space corresponding to highly redshifted near-coincident black holes. It is argued that one should consider, instead of $H$ eigenstates, eigenstates of $2 L_{0}=H+K$, where $K$ is the generator of special conformal transformations. The result is a well defined Hilbert space with a discrete spectrum describing the $N$-black hole dynamics.
\end{abstract}




\section{Contents}

1. Introduction 1

2. Derivation of the $D=5$ Multi-Black Hole Moduli Space 4

3. The Near-Horizon Limit 8

凹. Conformal Symmetry 10

5. Discussion 13

\section{Introduction}

One of the simplest examples of conformally-invariant quantum mechanics [1] is given by the single-particle Hamiltonian

$$
H=\frac{p^{2}}{2}+\frac{g}{2 x^{2}}
$$

This system has a generator of dilations

$$
D=\frac{1}{2}(p x+x p)
$$

and special conformal transformations

$$
K=\frac{x^{2}}{2}
$$

These operators obey the $S L(2, \mathbb{R})$ conformal algebra

$$
[D, H]=2 i H, \quad[D, K]=-2 i K, \quad[H, K]=-i D .
$$

Of course since $D$ and $K$ do not commute with the Hamiltonian, they are not symmetries of the theory in the usual sense. Rather $S L(2, \mathbb{R})$ can be used to relate states of different energies.

${ }^{*}$ After Sept. 1: New High Energy Theory Center; Rutgers University; Piscataway, NJ 08854. 
In fact the theory defined by $H$ is hard to make sense of because there is no ground state. The wave function wants to spread out to infinite $x$. Many years ago [1] de Alfaro, Fubini and Furlan (DFF) suggested that one should consider, instead of $H$ eigenstates, eigenstates of

$$
L_{0}=\frac{1}{2}(H+K)=\frac{p^{2}}{4}+\frac{g}{4 x^{2}}+\frac{x^{2}}{4} .
$$

$L_{0}$ has a well behaved discrete spectrum of normalizable eigenstates. ${ }^{1}$

This same problem reappeared recently in a new guise [2, 3, 4, 5]. Consider a charged BPS particle in a stationary trajectory a fixed but small distance from the horizon of an extremal Reissner-Nordström black hole. The near-horizon geometry of extremal Reissner-Nordström is $A d S_{2} \times S^{2}$, so in this region the particle follows a trajectory of constant $r$ in the $A d S_{2}$ coordinates

$$
d s^{2}=-r^{2} d t^{2}+\frac{d r^{2}}{r^{2}} .
$$

Non-relativistic radial motion of this particle is described by the Hamiltonian $H$ of equation (1.1) with $g=0 . g$ becomes nonzero when $S^{2}$ angular momentum is added. In this context the $S L(2, \mathbb{R})$ symmetry generated by $H, D$ and $K$ is nothing but the $S L(2, \mathbb{R})$ isometries of $A d S_{2}$.

The absence of a good ground state for $H$ in this context is the familiar statement that there is a divergent continuum of very low-energy states corresponding to highly redshifted trajectories very near the horizon at $r \rightarrow 0$. The origin of this problem is that the time conjugate to $H$ is not a good global time coordinate on $A d S_{2}$. On the other hand $L_{0}=\frac{1}{2}(H+K)$ is conjugate to a good global time coordinate. So, as pointed out in [2] and illustrated in figure [1, in the black hole context the DFF suggestion simply amounts to an improved choice of time coordinate.

In this paper we apply this philosophy to the problem of the quantum mechanics of $N$ slowly-moving five-dimensional BPS black holes, or equivalently quantum mechanics on the $N$-black hole moduli space. ${ }^{2}$ This moduli space has two types of noncompact regions into which the wave function spreads, preventing the existence of a normalizable ground state. The first such region is asymptotically flat $\left(\mathbb{R}^{4 N}\right.$ in five dimensions) corresponding to widely separated black holes. We show that in a low-energy, near-horizon limit, this noncompact region decouples from the near-horizon region of strongly interacting black holes. The decoupling of this near-horizon region suggests that quantum

\footnotetext{
${ }^{1}$ The spectrum is generated with $2 L_{ \pm 1}=H-K \mp i D$, which obey $\left[L_{1}, L_{-1}\right]=2 L_{0}$ and $\left[L_{0}, L_{ \pm 1}\right]=$ $\mp L_{ \pm 1}$.

${ }^{2}$ The $N=2$ case in four dimensions was analyzed in $[6]$.
} 


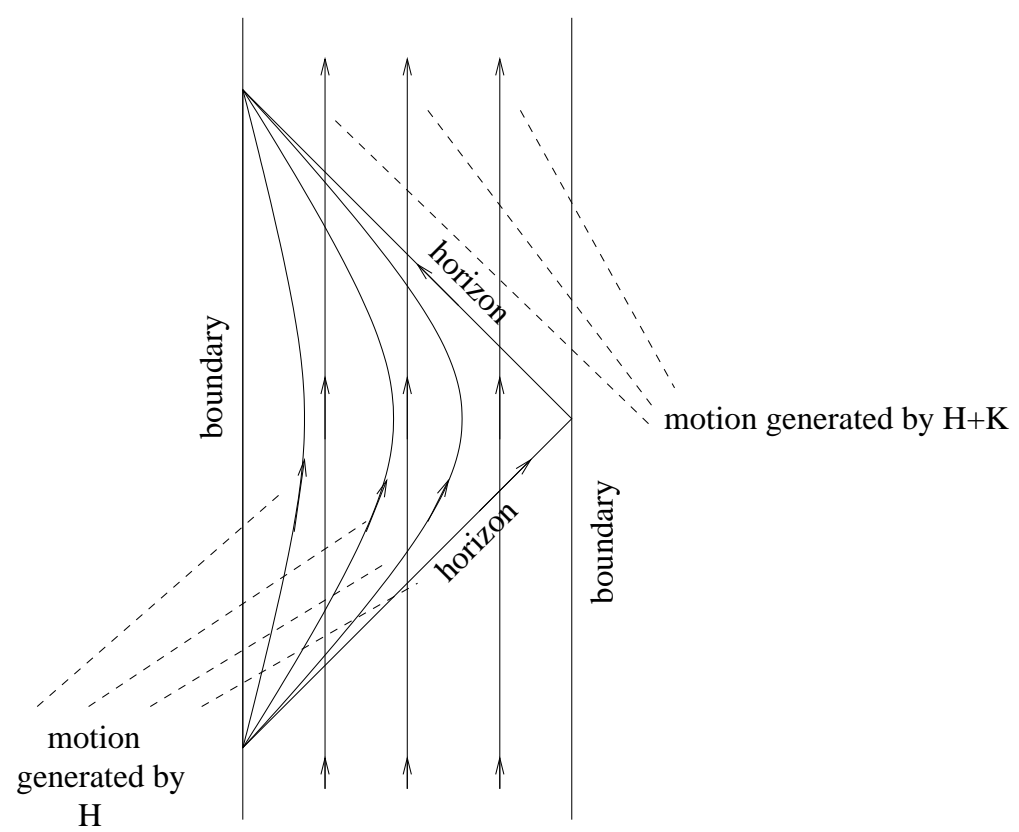

Figure 1: The $A d S_{2}$ time coordinate conjugate to $H$ is badly behaved at the horizon, but the time conjugate to $H+K$ is a good global coordinate.

states supported in the near-horizon region can be interpreted as internal states of a single composite black hole, and that the number of such states is related to the black hole entropy. However the quantum Hilbert space is again hard to make sense of because of noncompact regions of the moduli space corresponding to near-coincident black holes. The continuum of low-energy states again arise because of large redshifts for near-coincident black holes. For the two-black hole case $(N=2)$ the problem is equivalent to that of a BPS particle near the black hole horizon, and the noncompact region of the moduli space is exactly the troublesome $r \rightarrow 0$ region discussed above.

This noncompact region can be eliminated by a generalization of the DFF suggestion. We show that the near-horizon moduli space has an $S L(2, \mathbb{R}$ ) (more precisely $D(2,1 ; 0))$ symmetry. Trading $H$ for $H+K$, the special conformal generator $K$ acts as a potential on the moduli space which diverges in all the noncompact directions, preventing the indefinite spread of the quantum wave functions. We note that unlike the 2-body case, trading $H$ for $H+K$ cannot (as far as we know) be viewed as a new choice of time coordinate, and hence the $N$-body generalization is rather nontrivial. Nevertheless the result of the trade is a normalizable ground state and a well defined $4 N$-body quantum mechanics.

It is worth emphasizing that no general argument that we know of guaranteed the existence of an $S L(2, \mathbb{R})$ symmetry in the near-horizon limit. The existence of a 
dilational symmetry at low energies follows simply from dimensional arguments. The existence of a special conformal generator $K$ however requires a very particular form of the geometry.

The theory we specifically consider is five-dimensional $N=1$ (8 supercharges) supergravity with no vector multiplets. This can be obtained by compactification of $M$-theory on a Calabi-Yau with $b_{2}=1$. The black holes carry graviphoton charge. They can be described as M2-branes wrapping the Calabi-Yau 2-cycle. Other types of black holes - for example those appearing in theories with more supercharges or vector

multiplets - have a different structure typically with extra degrees of freedom. We do not know if the $S L(2, \mathbb{R})$ symmetry exists in all cases.

The outline of this paper is as follows. In section 2 we give a detailed derivation of the moduli space metric for $N$ BPS black holes in five dimensions, completing previous work on the subject [7, 8, 9, 10] and in particular exhibiting the requisite four supersymmetries. The problem of finding the supersymmetries in four dimensions remains unsolved (although see [10]). In section 3 we find the near-horizon limit of the moduli space geometry. Using results of [1], the $D(2,1 ; 0)$ conformal symmetry of the near-horizon moduli space quantum mechanics is displayed in section $₫$. Concluding comments on the possible role of this near-horizon quantum mechanics as a dual description of black holes, the relation to M-theory constructions and the $A d S / C F T$ correspondence [12], are in section 5.

\section{Derivation of the $D=5$ Multi-Black Hole Moduli Space}

In this section we derive the moduli space of $N$ BPS black holes in five-dimensional $\mathcal{N}=1$ supergravity with a single $U(1)$ charge, coupled to the graviphoton, and no vector multiplets. Neutral hypermultiplets, if present, do not affect the discussion because they decouple. The relevant part of the action is [13]

$$
S=\frac{M_{p}}{2} \int d^{5} x \sqrt{g}\left[M_{p}^{2} R-\frac{3}{4} F^{2}\right]+\frac{\lambda}{2} \int A \wedge F \wedge F
$$

where $\lambda=1$ (but we keep it general for most of the discussion) and we have normalized the vector field in an unconventional but convenient way.

The static, $N$ extremal black hole solutions are given by

$$
d s^{2}=-\psi^{-2} d t^{2}+\psi d \vec{x}^{2}
$$

and

$$
A=M_{p} \psi^{-1} d t
$$


where $\psi$ is the harmonic function on $\mathbb{R}^{4}$

$$
\psi=1+\sum_{A=1}^{N} \frac{Q_{A} L_{p}^{2}}{\left|\vec{x}-\vec{x}_{A}\right|^{2}}
$$

$L_{p}=\frac{1}{M_{p}}$ and $\vec{x}_{A}$ is the $\mathbb{R}^{4}$ coordinate of the $A$ th black hole with charge $Q_{A}$.

Supersymmetry $^{3}$ implies a no-force condition between the black holes, and thus the $\vec{x}_{A}$ are moduli for the set of states described by equations (2.2). The effective action governing slowly-moving black holes is therefore described by the metric on this moduli space; we will derive this metric following [14, 7 , 8], and show that (for $\lambda=1$ ) it has the properties expected for $\mathcal{N}=4 B$ supersymmetric quantum mechanics [10, [1].

The first step in the derivation of the effective action, following [14] is to add the source terms

$$
S_{\text {source }}=-\frac{6 \pi^{2}}{L_{p}} \sum_{A=1}^{N} Q_{A} \int d s_{A}+6 \pi^{2} \sum_{A=1}^{N} Q_{A} \int A_{\mu} d x_{A}^{\mu}
$$

to the action, where $d s_{A}$ is the line element of the center of the $A$ th black hole. In the remainder of this section we set $L_{p}=1$. The terms in equation (2.3) are necessary because the equations of motion following from (2.1) are not satisfied at the timelike singularities located behind the $N$ black hole event horizons at $\vec{x}=\vec{x}_{A}$. These source terms will affect the calculation as we have set it up, because we work in a gauge in which the zero modes corresponding to motion of the $A$ th black hole do not vanish at $\vec{x}=\vec{x}_{A}$. In principle it should be possible to choose a gauge in which the zero mode vanishes at $\vec{x}=\vec{x}_{A}$, and hence avoid adding the terms (2.3). However we have found the integrals most tractable in the chosen gauge. In [14] the moduli space metric (in the four-dimensional Einstein-Maxwell theory) was computed by first smoothing out these sources and then letting them approach delta functions at the end of the calculation. We shall not find this cumbersome procedure necessary.

An ansatz describing the perturbation of the black hole solution (2.2) by non-zero, but small velocities to linear order is

$$
d s^{2}=-\psi^{-2} d t^{2}+\psi d \vec{x}^{2}+2 \psi^{-2} \vec{R} \cdot d \vec{x} d t
$$

and

$$
A=\psi^{-1} d t+\left(\vec{P}-\psi^{-1} \vec{R}\right) \cdot d \vec{x}
$$

\footnotetext{
${ }^{3}$ Actually, this is more general than supersymmetry, since it holds for any value of $\lambda$ in the action.
} 
where $\vec{P}$ and $\vec{R}$ are quantities that are first order in velocities. Also, in equation (2.2C), $\vec{x}_{A}$ is replaced with $\vec{x}_{A}+\vec{v}_{A} t$. Note that this is the most general galilean-invariant ansatz to linear order.

Inserting the ansatz (2.4) into equation (2.3) gives, keeping only terms up to second order in velocities,

$$
\begin{aligned}
S_{\text {source }}= & -6 \pi^{2} \sum_{A=1}^{N} Q_{A} \int d t \psi^{-1}\left(1-\vec{R} \cdot \vec{v}_{A}-\frac{1}{2} \psi^{3} \vec{v}_{A}^{2}\right) \\
& +6 \pi^{2} \sum_{A=1}^{N} Q_{A} \int d t\left(\psi^{-1}+\vec{P} \cdot \vec{v}_{A}-\psi^{-1} \vec{R} \cdot \vec{v}_{A}\right) \\
= & \frac{1}{2} \sum_{A=1}^{N} Q_{A} \int d t\left(6 \pi^{2} \psi^{2} \vec{v}_{A}^{2}+12 \pi^{2} \vec{v}_{A} \cdot \vec{P}\right) .
\end{aligned}
$$

The total action, (2.1) and (2.3) is, to second order in velocities,

$$
\begin{aligned}
& S=\frac{1}{2} \int d^{5} x\left\{6 \pi^{2} \psi^{2} \sum_{A=1}^{N} Q_{A} \delta^{(4)}\left(\vec{x}-\left(\vec{x}_{A}+\vec{v}_{A} t\right)\right) \vec{v}_{A}^{2}+12 \pi^{2} \sum_{A=1}^{N} Q_{A} \delta^{(4)}\left(\vec{x}-\left(\vec{x}_{A}+\vec{v}_{A} t\right)\right) \vec{v}_{A} \cdot \vec{P}\right. \\
& +3 \partial_{t} \vec{P} \cdot \vec{\partial} \psi-\frac{3}{4} \psi^{-1}\left(\partial_{i} P_{j}-\partial_{j} P_{i}\right)^{2}+3 \psi^{-2}\left(\partial_{i} P_{j}-\partial_{j} P_{i}\right) \partial_{i} R_{j}-\frac{1}{2} \psi^{-3}\left(\partial_{i} R_{j}-\partial_{j} R_{i}\right)^{2} \\
& \left.-3 \psi\left(\partial_{t} \psi\right)^{2}-3 \lambda \psi^{-1} \epsilon^{i j k l} \partial_{i} P_{k} \partial_{j} P_{l}+3 \lambda \psi^{-2} \epsilon^{i j k l} \partial_{i} P_{k} \partial_{j} R_{l}-\lambda \psi^{-3} \epsilon^{i j k l} \partial_{i} R_{k} \partial_{j} R_{l}+\text { t.d. }\right\}
\end{aligned}
$$

where $i, j=1, \ldots, 4$ are spatial indices and t.d. stands for total derivative.

Our immediate goal is to eliminate $\vec{P}$ and $\vec{R}$ from the effective action (2.6) to get an action involving only $\vec{v}_{A}$ as a dynamical variable. This requires the equations of motion which yield

$$
\begin{gathered}
\vec{\partial}^{2} \psi=-4 \pi^{2} \sum_{A=1}^{N} Q_{A} \delta^{(4)}\left(\vec{x}-\vec{x}_{A}-\vec{v}_{A} t\right), \\
d R=-3 \psi^{2} \sum_{A=1}^{N} d \frac{Q_{A}}{\left|\vec{x}-\left(\vec{x}_{A}+\vec{v}_{A} t\right)\right|^{2}} \wedge v_{A},
\end{gathered}
$$

and

$$
d P=-2 \psi \sum_{A=1}^{N} d \frac{Q_{A}}{\left|\vec{x}-\left(\vec{x}_{A}+\vec{v}_{A} t\right)\right|^{2}} \wedge v_{A} .
$$


Equation (2.7a) follows from the $A_{0}$ (and $g_{00}$ ) equation of motion and is solved by equation (2.2c). Equations (2.7b) and (2.7b) follow from linear combinations of the $g_{0 i}$ and $A_{i}$ equations of motion.

Note that $\vec{P}$ and $\vec{R}$ appear in the effective action (2.6) only as $d P$ and $d R$, except in the second and third terms. In order to apply equations (2.7), we need to do the following manipulation on those terms:

$$
\begin{aligned}
& 12 \pi^{2} \sum_{A=1}^{N} Q_{A} \delta^{(4)}\left(\vec{x}-\left(\vec{x}_{A}+\vec{v}_{A} t\right)\right) \vec{v}_{A} \cdot \vec{P}+3 \partial_{t} \vec{P} \cdot \vec{\partial} \psi \\
& \quad=-3 \vec{P} \cdot \vec{\partial} \partial_{t} \psi-3 \sum_{A=1}^{N} \vec{\partial}^{2} \frac{Q_{A}}{\left|\vec{x}-\left(\vec{x}_{A}+\vec{v}_{A} t\right)\right|^{2}} \vec{v}_{A} \cdot \vec{P}+\mathrm{t} . \mathrm{d} . \\
& \quad=3 \vec{P} \cdot \vec{\partial} \sum_{A=1}^{N} \vec{v}_{A} \cdot \vec{\partial} \frac{Q_{A}}{\left|\vec{x}-\left(\vec{x}_{A}+\vec{v}_{A} t\right)\right|^{2}}-3 \sum_{A=1}^{N} \vec{\partial}^{2} \frac{Q_{A}}{\left|\vec{x}-\left(\vec{x}_{A}+\vec{v}_{A} t\right)\right|^{2}} \vec{v}_{A} \cdot \vec{P}+\mathrm{t} . \mathrm{d} . \\
& \quad=-3 \sum_{A=1}^{N} \vec{v}_{A} \cdot \vec{\partial} \vec{P} \cdot \vec{\partial} \frac{Q_{A}}{\left|\vec{x}-\left(\vec{x}_{A}+\vec{v}_{A} t\right)\right|^{2}}+3 \sum_{A=1}^{N} \vec{\partial} \frac{Q_{A}}{\left|\vec{x}-\left(\vec{x}_{A}+\vec{v}_{A} t\right)\right|^{2}} \cdot \vec{\partial} \vec{P} \cdot \vec{v}_{A}+\mathrm{t} . \mathrm{d} . \\
&=-3 \sum_{A=1}^{N} v_{A i}\left(\partial_{i} P_{j}-\partial_{j} P_{i}\right) \partial_{A j} \psi+\mathrm{t} . \mathrm{d} .
\end{aligned}
$$

Now the only $P$-dependence of the action is in the form $d P$, and we can apply equations (2.7). This yields

$$
\begin{aligned}
S=\frac{1}{2} \int & d^{5} x\left\{-\frac{3}{2} \psi^{2} \sum_{A=1}^{N} \vec{\partial}_{A}^{2} \psi \vec{v}_{A}^{2}-3 \psi \sum_{A, B=1}^{N} \vec{v}_{A} \cdot \vec{v}_{B} \vec{\partial}_{A} \psi \cdot \vec{\partial}_{B} \psi+3 \psi \sum_{A, B=1}^{N} \vec{v}_{A} \cdot \vec{\partial}_{B} \psi \vec{v}_{B} \cdot \vec{\partial}_{A} \psi\right. \\
& \left.-3 \psi \sum_{A, B=1}^{N} \vec{v}_{A} \cdot \vec{\partial}_{A} \psi \vec{v}_{B} \cdot \vec{\partial}_{B} \psi-3 \lambda \psi \epsilon^{i j k l} \sum_{A, B=1}^{N} \partial_{A i} \psi v_{A j} \partial_{B k} \psi v_{B l}+\text { t.d. }\right\}
\end{aligned}
$$

or equivalently

$$
S=-\frac{1}{4} \int d t \sum_{A, B=1}^{N}\left(\delta^{i j} \delta_{k l}+\delta_{k}^{i} \delta_{l}^{j}-\delta_{l}^{i} \delta_{k}^{j}+\lambda \epsilon^{i j}{ }_{k l}\right) \partial_{A i} \partial_{B j}\left(\int d^{4} x \psi^{3}\right) v^{A k} v^{B l}
$$

Precisely in the supersymmetric case, when $\lambda=1,{ }^{4}$ this can be rewritten as

$$
S=\frac{1}{4} \int d t v^{A k} v^{B l}\left(\delta_{k}^{i} \delta_{l}^{j}+\sum_{r=1}^{3} I_{k}^{r i} I_{l}^{r j}\right) \partial_{A i} \partial_{B j} L
$$

\footnotetext{
${ }^{4} \lambda=-1$ differs by a reflection, and so we also get a supersymmetric moduli space with the antiselfdual complex structures.
} 
where

$$
L=-\int d^{4} x \psi^{3}
$$

and $I^{r}, r=1,2,3$ are the natural triplet of self-dual complex structures on $\mathbb{R}^{4}$. In [11] it was shown that any action of the form (2.11) has (after appropriately adding fermions) $\mathcal{N}=4 B$ supersymmetry (which has an $S U(2) R$-symmetry under which the bosons are complex doublets) for any function $L$. Hence we conclude that the multi-black hole moduli space has $\mathcal{N}=4 B$ supersymmetry.

We note that the first term of equation (2.10) (i.e. the term with $\delta^{i j} \delta_{k l}$ ) has previously appeared in the literature [7, 8] on the five-dimensional black hole moduli space, but the last three terms (required by supersymmetry) were not previously included.

\section{The Near-Horizon Limit}

In this section we describe the "near-horizon limit" of a collection of $N$ black holes, in which

$$
\frac{\left|\vec{x}_{A}-\vec{x}_{B}\right|^{2}}{L_{p}^{2}} \rightarrow 0
$$

with charges of order one. This corresponds to taking the characteristic distances between the black holes to be much less than the Planck length. It also corresponds to kinetic energies much less than the Planck mass, because the relative kinetic energy of two black holes becomes highly redshifted when they are near one another.

The limiting geometry can be derived by defining new coordinates

$$
\vec{U}_{A}=\frac{\vec{x}_{A}}{L_{p}^{3 / 2}}, \quad \vec{U}=\frac{\vec{x}}{L_{p}^{3 / 2}},
$$

and then taking

$$
L_{p} \rightarrow 0 .
$$

The spacetime metric for the $N$-black hole solution then becomes

$$
\frac{d s^{2}}{L_{p}^{2}}=-\psi^{-2} d t^{2}+\psi d \vec{U}^{2}
$$

with

$$
\psi=\sum_{A=1}^{N} \frac{Q_{A}}{\left|\vec{U}-\vec{U}_{A}\right|^{2}}
$$


(a)

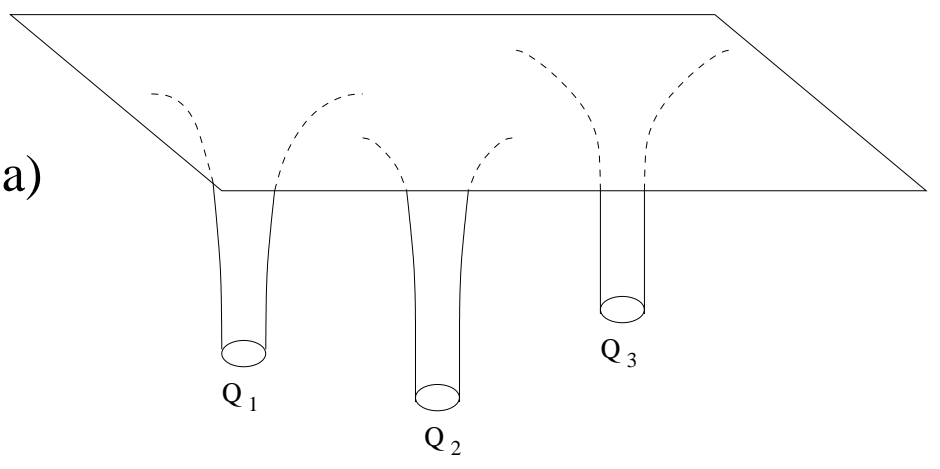

(c)

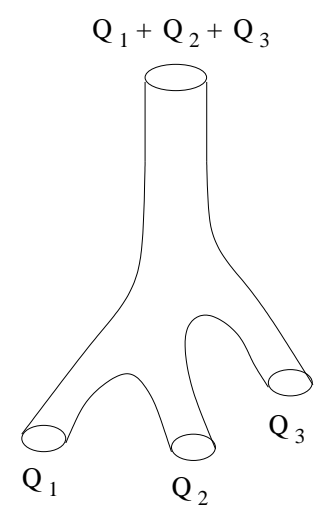

(b)

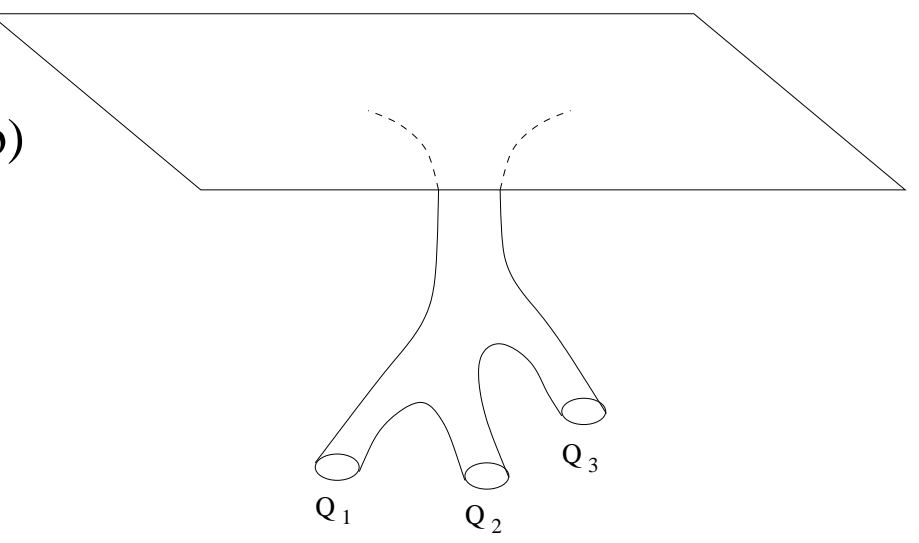

Figure 2: (a) Widely separated black holes. (b) Near-coincident black holes. (c) The nearhorizon limit.

An illustration of the resultant spatial geometry at a moment of fixed time is given in figure 2 for $N=3$. Before the limit is taken (figure 2a), the geometry has an asymptotically flat region at large $|\vec{x}|$. Near the limit (figure 2b), as the origin is approached along a spatial trajectory, a single "throat" approximating that of a charge $\sum Q_{A}$ black hole is encountered. This throat region is an $A d S_{2} \times S^{3}$ geometry with radii of order $\sqrt{\sum Q_{A}}$. As one moves deeper inside the throat towards the horizon, the throat branches into smaller throats, each of which has smaller charge and correspondingly smaller radii. Eventually there are $N$ branches with charge $Q_{A}$. At the end of each of these branches is an event horizon. When the limit is achieved (figure 20c), the asymptotically flat region moves off to infinity. Only the charge $\sum Q_{A}$ "trunk" and the many branches remain.

It is also of interest to consider the near-horizon limit of the moduli space geometry. 

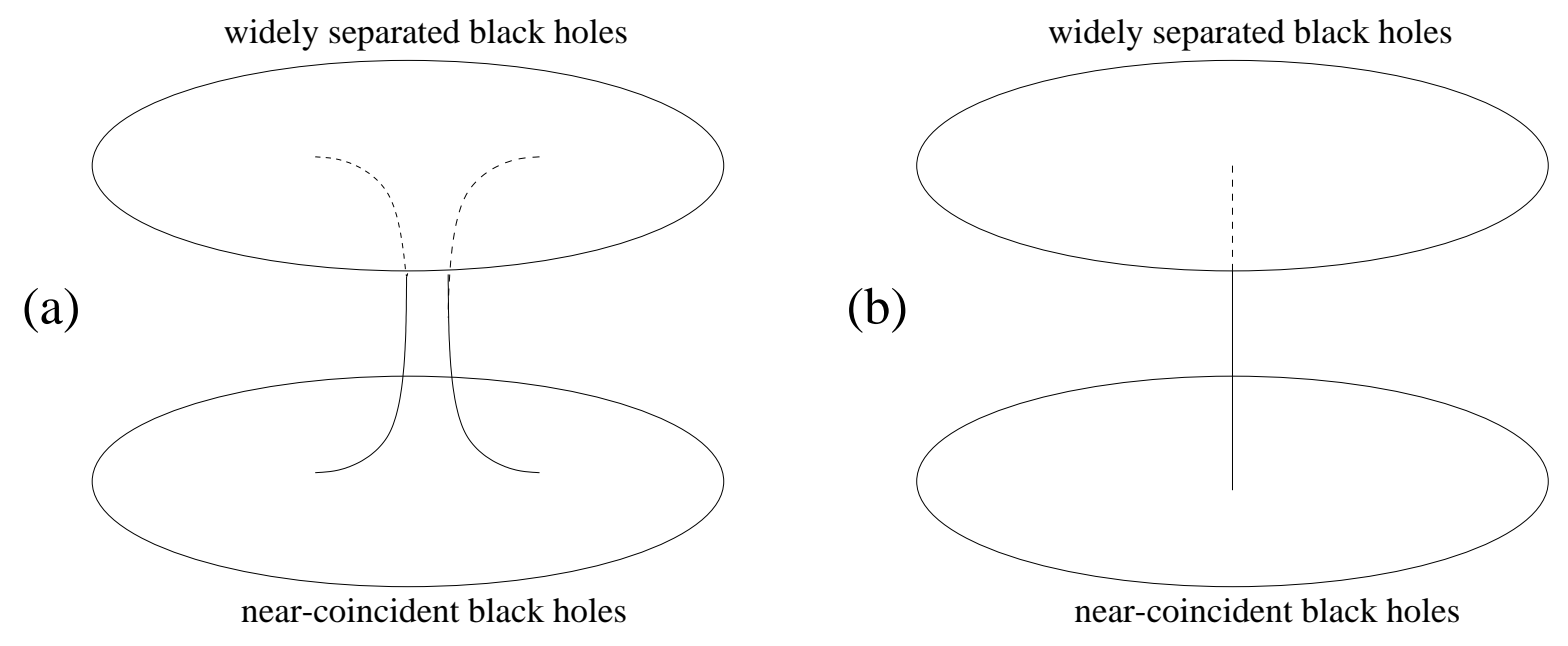

Figure 3: (a) Regions of the two-black hole moduli space. (b) The near-horizon limit.

This is simply ${ }^{5}$

$$
\frac{d s^{2}}{L_{p}^{3}}=\frac{1}{4}\left(\delta_{k}^{i} \delta_{l}^{j}+\sum_{r=1}^{3} I_{k}^{r i} I_{l}^{r j}\right)\left(\partial_{A i} \partial_{B j} L\right) d U^{A k} d U^{B l}
$$

with

$$
L=-\int d^{4} U \psi^{3}
$$

and $\psi$ now given by (3.5). This is illustrated in figure 3 for the case of $N=2$. Near the limit there is an asymptotically flat $\mathbb{R}^{4 N}$ region corresponding to all $N$ black holes widely separated. This is connected to the near-horizon region where the black holes are strongly-interacting, by tube-like regions which become longer and thinner as the limit is approached. When the limit is achieved, the near-horizon region is severed from the tubes and the asymptotically flat region. The near-horizon metric (3.6) develops four zero eigenvectors $U^{A k}=T^{k}$ corresponding to the decoupling of the four overall center of mass coordinates. For the case of two black holes the near-horizon region is just flat $\mathbb{R}^{4}$, with the tube touching at the origin.

\section{Conformal Symmetry}

The fact that the near-horizon moduli space geometry decouples from the rest of the moduli space, suggests that the quantum states on this moduli space may be interpreted as internal states of a single charge $\sum Q_{A}$ black hole. However an obstacle

\footnotetext{
${ }^{5}$ If the black holes are identical, one must take a quotient with respect to the permutation group.
} 
is immediately encountered in attempting to make sense of the quantum mechanics: the Hamiltonian has no ground state because of the infinite volume ascribed to nearcoincident black holes. Indeed, near $\vec{U}_{A}=\vec{U}_{B}$ the metric has a term

$$
\left(Q_{A}^{2} Q_{B}+Q_{B}^{2} Q_{A}\right) \frac{\left|d \vec{U}_{A}-d \vec{U}_{B}\right|^{2}}{\left|\vec{U}_{A}-\vec{U}_{B}\right|^{4}} .
$$

Defining $\vec{W}_{A B}=\frac{\vec{U}_{A}-\vec{U}_{B}}{\left|\vec{U}_{A}-\vec{U}_{B}\right|^{2}}$, (4.1) becomes

$$
\left(Q_{A}^{2} Q_{B}+Q_{B}^{2} Q_{A}\right)\left|d \vec{W}_{A B}\right|^{2} .
$$

This is just the flat metric on $\mathbb{R}^{4}$, with infinity corresponding to the near-coincident limit (and the origin to infinite separation). Because of these infinite-volume regions, the quantum system has an infinite number of states below any finite excitation energy, and no normalizable ground state. This is in conflict with the usual stringy picture of a black hole as a system with a gap and a discrete spectrum.

In this section we will show that the near-horizon quantum mechanics has an $S L(2, \mathbb{R})$ conformal symmetry (in fact a $D(2,1 ; 0)$ superconformal symmetry) with generators of time translations $H$, dilations $D$ and special conformal transformations $K$. The Hilbert space can then be sensibly defined as the eigenstates of $H+K$, rather than of $H$ itself.

To see this conformal symmetry we begin by dividing the potential $L$ appearing in the metric (3.6) up into pieces which represent the 1-body, 2-body and 3-body interactions

$$
\begin{gathered}
L_{1}=-\sum_{A=1}^{N} \int d^{4} U \frac{Q_{A}^{3}}{\left|\vec{U}-\vec{U}_{A}\right|^{6}}, \\
L_{2}=-3 \sum_{A \neq B}^{N} \int d^{4} U \frac{Q_{A}^{2} Q_{B}}{\left|\vec{U}-\vec{U}_{A}\right|^{4}\left|\vec{U}-\vec{U}_{B}\right|^{2}}, \\
L_{3}=-\sum_{A \neq B \neq C}^{N} \int d^{4} U \frac{Q_{A} Q_{B} Q_{C}}{\left|\vec{U}-\vec{U}_{A}\right|^{2}\left|\vec{U}-\vec{U}_{B}\right|^{2}\left|\vec{U}-\vec{U}_{C}\right|^{2}},
\end{gathered}
$$

with $L=L_{1}+L_{2}+L_{3}$. It is a curious (and unexplained) fact that there are no 4-body or higher interactions. $L_{1}$ is a divergent $\vec{U}_{A}$-independent constant. This divergence does not enter the metric which involves only derivatives of $L$. Hence $L_{1}$ can be ignored. 
$L_{2}$ has logarithmic divergences near $\vec{U}=\vec{U}_{A}$. With a cutoff $\delta$ the divergences go like $\frac{\ln \delta}{\left|\vec{U}_{A}-\vec{U}_{B}\right|^{2}}$. These terms also disappear after differentiating $L$ to form the metric. The remaining term, which does contribute to the metric, is

$$
L_{2}=-6 \pi^{2} \sum_{A \neq B}^{N} \frac{Q_{A}^{2} Q_{B} \ln \left|\vec{U}_{A}-\vec{U}_{B}\right|}{\left|\vec{U}_{A}-\vec{U}_{B}\right|^{2}} .
$$

$L_{3}$ is finite and can be defined without a regulator. It follows that (unlike $L_{2}$ ) it is a homogeneous polynomial of degree -2 obeying

$$
U^{A i} \partial_{A i} L_{3}=-2 L_{3}
$$

We further note that by rotational invariance

$$
U^{A i} I_{i}^{r j} \partial_{A j} L_{2}=0=U^{A i} I_{i}^{r j} \partial_{A j} L_{3} .
$$

First we look for a dilational symmetry of the 2-body and 3-body metrics $g_{2}$ and $g_{3}$. It is easy to see that the vector field

$$
D^{A i} \partial_{A i}=-U^{A i} \partial_{A i}
$$

generates a dilational symmetry under which the metric transforms as $\mathcal{L}_{D} g_{a b}=2 g_{a b}$. Although $L_{2}$ (equation (4.4) is not homogeneous because of the logarithm, the anomalous piece does not contribute in the metric.

It was shown in [11] that in order to have a special conformal symmetry the oneform

$$
D_{A i} d U^{A i}
$$

must be closed. The one-form is obtained from the vector field by lowering the index with the metric $g=g_{2}+g_{3}$. A little algebra using equation (3.6) reveals that any metric constructed from a potential obeying the weight -2 scaling relation (4.5) has $U^{A i}$ as a zero eigenvector [11]. Therefore

$$
D_{3 A i}=-g_{3 A i B j} U^{B j}=0 .
$$

Explicit computation then gives (suppressing factors of $L_{p}$ )

$$
D_{A i} d U^{A i}=-g_{2 A i B j} U^{B j} d U^{A i}=d\left[6 \pi^{2} \sum_{A \neq B}^{N} \frac{Q_{A}^{2} Q_{B}}{\left|\vec{U}_{A}-\vec{U}_{B}\right|^{2}}\right] .
$$


Hence $D_{A i} d U^{A i}$ is closed, and as shown in [11], the quantity in square brackets is the generator of special conformal transformations

$$
K=6 \pi^{2} \sum_{A \neq B}^{N} \frac{Q_{A}^{2} Q_{B}}{\left|\vec{U}_{A}-\vec{U}_{B}\right|^{2}}
$$

This geometry meets all the criteria described in [11] for the full $D(2,1 ; 0)$ superconformal symmetry. This group is the special case of the $D(2,1 ; \alpha)$ superconformal groups for which there is an $S U(1,1 \mid 2)$ subgroup. ${ }^{6}$ The appearance of $S U(1,1 \mid 2)$, in the nearhorizon limit of the spacetime geometry of a single black hole, was observed in [15].

It is worth noting that while the existence of the dilational symmetry was more or less guaranteed by the nature of the near-horizon limit, the same cannot be said for the special conformal symmetry. Indeed if the coefficients of the four terms in (2.10) are perturbed, the dilational symmetry remains but the special conformal symmetry generically disappears.

\section{Discussion}

We have given a manifestly supersymmetric presentation of quantum mechanics on the five-dimensional $N$-black hole moduli space. At low energies, the dynamics of nearcoincident black holes decouples from that of widely separated black holes, and an enhanced $S L(2, \mathbb{R})$ symmetry appears. There are noncompact regions of this near-horizon moduli space corresponding to coincident black holes. These regions are eliminated by the potential $K$ in the modified Hamiltonian $L_{0}=\frac{1}{2}(H+K)$, which is singular at the boundary of the noncompact regions. We then have an apparently well defined, albeit complicated, quantum mechanics describing black holes which are neither widely separated nor coincident. A detailed description of the quantum states of this system remains to be found. For now we content ourselves with a few comments on their possible interpretation.

Let us return to the case of $M$-theory on Calabi-Yau with $b_{2}=1$. An alternate picture of the charge $N$ black hole is then an $M 2$-brane holomorphically wrapped $N$ times around the Kähler class. In the quantum theory one has a quantum mechanics on the moduli space of $N$-wrapped holomorphic cycles. The black hole entropy should be given by the logarithm of the number of ground states of the $M 2$-brane, which is roughly speaking the Euler character of the moduli space. While $A d S_{2} / C F T_{1}$ duality [12, 16, 17, 18, 19, 4, 20, 21, 22] has eluded a proper understanding, this quantum

\footnotetext{
${ }^{6}$ Specifically, $D(2,1 ; 0)$ is the semi-direct sum of $S U(1,1 \mid 2)$ and $S U(2)$.
} 
mechanics on the $M 2$-brane moduli space should more or less be the theory which lives on the boundary (boundaries?) of $A d S_{2}$.

This moduli space has several components. In one component-which we will refer to as the "Higgs" branch in an abuse of language (since there is no gauge theory on the M2-brane) - one has a single connected M2-brane mapped holomorphically into the Calabi-Yau. There is one center of mass degree of freedom corresponding to transverse motion on the spatial $\mathbb{R}^{4}$. The quantum states on the analog of this branch for 3branes on $K^{3} \times S^{1}$ and 5-branes on Calabi-Yau $\times S^{1}$ were successfully related to black hole entropy in [23] and 24]. ${ }^{7}$ In addition there are "Coulomb" branches corresponding to disconnected membranes. These branches have up to $N$ center-of-mass coordinates. Since we have only a single type of charge there is no way to lift these branches. The importance of these branches for understanding $A d S_{2}$ black holes was discussed in [17.

Naïvely what has been described in this paper has $N$ center-of-mass coordinates and is therefore the Coulomb branch. In analogy with the calculations in [23, 24] one might expect that the black hole entropy counts states on the Higgs branch, and so should not be captured by the Coulomb branch considerations of this paper. However we would like to suggest an alternate interpretation. The Coulomb branch described herein divides into the far-Coulomb branch (containing widely separated black holes) and the near-Coulomb branch (containing near-coincident black holes). As we have seen, these two regions decouple completely at low energies. A natural conjecture is that the nearCoulomb branch is in fact a dual description of the Higgs branch, described above as the quantum mechanics of a single $N$-wrapped membrane. A related phenomenon occurs in the D1-D5 black hole, for which the near-Coulomb branch is a dual description of the small-instanton region of the Higgs branch - for a recent discussion, see [25]. If this is the case one does expect quantum mechanics in the geometry (3.6) to capture the black hole entropy.

\section{Acknowledgments}

We are grateful to R. Britto-Pacumio, J. Maldacena, M. Spradlin, P. Townsend and especially G. Papadapolous for useful conversations. This work was supported in part by an NSERC PGS B Scholarship and DOE grant DE-FG02-91ER40654.

\footnotetext{
${ }^{7}$ Note however that these computations of the entropy required only the dimension of the moduli space, while membranes on a Calabi-Yau require the cohomology of the moduli space. This is much more difficult to compute, and to date there is no microscopic computation of the entropy of these black holes.
} 


\section{References}

[1] V. de Alfaro, S. Fubini and G. Furlan, "Conformal Invariance in Quantum Mechanics," Nuovo Cim. 34A (1976) 569.

[2] P. Claus, M. Derix, R. Kallosh, J. Kumar, P. K. Townsend and A. van Proeyen, "Black Holes and Superconformal Mechanics," Phys. Rev. Lett. 81 (1998) 4553; hep-th/9804177.

[3] J. A. de Azcárraga, J. M. Izquerido, J. C. Pérez-Bueno and P. K. Townsend, "Superconformal Mechanics, Black Holes and Non-linear Realizations," Phys. Rev. D 59 (1999) 084015; hep-th/9810230.

[4] G. W. Gibbons and P. K. Townsend, "Black Holes and Calogero Models," Phys. Lett. B 454 (1999) 187; hep-th/9812034.

[5] R. Kallosh, "Black Holes and Quantum Mechanics," hep-th/9902007.

[6] J. Traschen and R. Ferrell, "Quantum Mechanical Scattering of Charged Black Holes," Phys. Rev. D 45 (1992) 2628; hep-th/9205061.

[7] K. Shiraishi, "Moduli Space Metric for Maximally-Charged Dilaton Black Holes," Nucl. Phys. B 402 (1993) 399.

[8] D. M. Kaplan and J. Michelson, "Scattering of Several Multiply Charged Extremal $D=5$ Black Holes," Phys. Lett. B 410 (1997) 125; hep-th/9707021.

[9] G. W. Gibbons and R. Kallosh, "Topology, Entropy and Witten Index of Dilaton Black Holes," Phys. Rev. D 51 (1995) 2839; hep-th/9407118.

[10] G. W. Gibbons, G. Papadopoulos and K. S. Stelle, "HKT and OKT Geometries on Soliton Black Hole Moduli Spaces," Nucl. Phys. B 508 (1997) 623; hep-th/9706207.

[11] J. Michelson and A. Strominger, "The Geometry of (Super) Conformal Quantum Mechanics," HUTP-99/A045, hep-th/9907191.

[12] J. Maldacena, "The Large N Limit of Superconformal Field Theories and Supergravity," Adv. Theor. Math. Phys. 2 (1998) 231; hep-th/9711200.

[13] E. Cremmer, "Supergravities in 5 Dimensions," in Superspace and Supergravity, Eds. S. W. Hawking and M. Roček (Cambridge University Press, 1981) 267.

[14] R. Ferrell and D. Eardley, "Slow-Motion Scattering and Coalescence of Maximally Charged Black Holes," Phys. Rev. Lett. 59 (1987) 1617.

[15] J. P. Gauntlett, R. C. Myers and P. K. Townsend, "Black Holes of $D=5$ Supergravity," Class. and Quant. Grav. 16 (1999) 1. 
[16] A. Strominger, "AdS $S_{2}$ Quantum Gravity and String Theory," J. High Energy Phys. 01 (1999) 007; hep-th/9809027.

[17] J. Michelson, J. Maldacena and A. Strominger, "Anti-de Sitter Fragmentation," J. High Energy Phys. 02 (1999) 011; hep-th/9812073.

[18] T. Nakatsu and N. Yokoi, "Comments on Hamiltonian Formalism of AdS/CFT Correspondence," Mod. Phys. Lett. A 14 (1999) 147; hep-th/9812047

[19] M. Cadoni and S. Mignemi, "Asymptotic Symmetries of $A d S_{2}$ and Conformal Group in $d=1, "$ INFNCA-TH9901, hep-th/9902040.

[20] P. K. Townsend, "M(atrix) Model/AdS $S_{2}$ Correspondence," DAMTP-1999-33, hep-th/9903043.

[21] M. Spradlin and A. Strominger, "Vacuum States for $A d S_{2}$ Black Holes," HUTP-99/A014, hep-th/9904143.

[22] J. Blum, "Supersymmetric Quantum Mechanical Description of Four Dimensional Black Holes," LBNL-43734, hep-th/9907101.

[23] A. Strominger and C. Vafa, "Microscopic Origin of the Bekenstein-Hawking Entropy," Phys. Lett. B 379 (1996) 99; hep-th/9601029.

[24] J. Maldacena, A. Strominger and E. Witten, "Black Hole Entropy in M-Theory," J. High Energy Phys. 12 (1997) 002; hep-th/9711053.

[25] M. Berkooz and H. Verlinde, "Matrix Theory, AdS/CFT and Higgs-Coulomb Equivalence," IASSNS-HEP-99/67, PUPT-1879, hep-th/9907100. 